\title{
Combining node location and node ratio as a prognostic factor for surgical resected non-small cell lung cancer: a population-based study
}

\author{
Huajie Xing ${ }^{1 \#}$, Mengyu Hu${ }^{2 \#}$, Jingyu Chen ${ }^{1}$, Yongqing Guo ${ }^{1}$, Deruo Liu ${ }^{1}$, Chaoyang Liang ${ }^{1}$ \\ ${ }^{1}$ Department of Thoracic Surgery, China-Japan Friendship Hospital, Beijing, China; ${ }^{2}$ Department of Breast Oncology, Beijing Cancer Hospital, \\ Beijing, China \\ Contributions: (I) Conception and design: H Xing, C Liang; (II) Administrative support: J Chen, Y Guo; (III) Provision of study materials or patients: \\ M Hu, D Liu; (IV) Collection and assembly of data: H Xing, M Hu; (V) Data analysis and interpretation: H Xing, M Hu; (VI) Manuscript writing: \\ All authors; (VII) Final approval of manuscript: All authors. \\ \#These authors contributed equally to this work. \\ Correspondence to: Chaoyang Liang, MD. Department of Thoracic Surgery, China-Japan Friendship Hospital, Chaoyang District, Beijing 100032, \\ China. Email: zryhyyxwk@sina.com.
}

\begin{abstract}
Background: In the TNM system only the anatomic location is used to define nodal status. In this study we aim to evaluate the effectiveness of combining the location and ratio of metastatic lymph node (pN-NR) for the prognosis of non-small cell lung cancer (NSCLC).

Methods: Patients with pN1/pN2 NSCLC were retrieved from the SEER database. The optimal cut point of NR was determined with the maximal selecting test. All patients were divided into 4 categories with combination of $\mathrm{pN}$ ( $\mathrm{pN} 1$ or $\mathrm{pN} 2$ ) and $\mathrm{NR}$ (low or high). The $\mathrm{pN}-\mathrm{NR}$ was investigated as a predictor of overall survival (OS) and cause-specific survival (CSS) using Cox regression models. Survival curves were plotted using the Kaplan-Meier method and the difference was compared with log-rank test.

Results: A total of 12,170 patients were enrolled. The optimal cut point of NR was 0.3. Patients were divided into 4 groups: pN1-NR $<0.3$, pN1-NR $\geq 0.3$, pN2-NR $<0.3$ and $\mathrm{pN} 2-\mathrm{NR} \geq 0.3$. The $\mathrm{pN}-\mathrm{NR}$ was an independent prognostic factor for survival. Compared with pN1-NR $<0.3$, the hazard ratio of OS was 1.405 (95\% CI: 1.295-1.524), 1.183 (95\% CI: 1.113-1257) and 1.717 (95\% CI: 1.607-1.835) times higher for $\mathrm{pN} 1-\mathrm{NR} \geq 0.3, \mathrm{pN} 2-\mathrm{NR}<0.3$ and $\mathrm{pN} 2-\mathrm{NR} \geq 0.3$ group, respectively. The survival curves of OS separated well between the $4 \mathrm{pN}-\mathrm{NR}$ groups, with 5 -year OS $47.1 \%$ for $\mathrm{pN} 1-\mathrm{NR}<0.3,43.0 \%$ for $\mathrm{pN} 2-\mathrm{NR}<0.3$, $35.0 \%$ for $\mathrm{pN} 1-\mathrm{NR} \geq 0.3$ and $28.5 \%$ for $\mathrm{pN} 2-\mathrm{NR} \geq 0.3$, and the $\mathrm{P}$ value between neighboring curves was statistically significantly. The same trend was observed for CSS. Subgroup analysis revealed similar results except the pneumonectomy group.
\end{abstract}

Conclusions: pN-NR could be a good predictor for the prognosis of NSCLC.

Keywords: Non-small cell lung cancer (NSCLC); lymph node ratio; prognosis

Submitted Feb 04, 2020. Accepted for publication Jun 11, 2020.

doi: $10.21037 /$ jtd-20-758

View this article at: http://dx.doi.org/10.21037/jtd-20-758

\section{Introduction}

Non-small cell lung cancer (NSCLC) is the leading cause of cancer-related death worldwide (1). For NSCLC patients who received radical surgical resection (lobectomy/ bilobectomy/pneumonectomy and LN dissection), the status of lymph node (LN) has always been an important prognostic factor (2). The tumor-node-metastasis (TNM) staging is a widely used classification system to predict the outcome of NSCLC. But in the TNM system only the anatomic location of metastatic LN is used to define the node status (3). 
Like in other solid organ malignant disease such as esophagus and breast, the number of metastatic LN has been proposed as a prognostic factor for NSCLC (4-6). However, the number of metastatic $\mathrm{LN}$ is confounded by the number of resected LN. In cases where few nodes were removed, the number of metastatic $\mathrm{LN}$ could not be accurately classified. To improve the prognostic system, the ratio between the metastatic $\mathrm{LN}$ and the resected $\mathrm{LN}$ (the $\mathrm{LN}$ ratio, NR), which takes into account not only the number of positive $\mathrm{LN}$ but also the number of LN harvested, removed the variability in nodal assessment to some extent. In recent years, there is increasing evidence indicating the NR to be significant prognostic factors for NSCLC (7-10). However, either the TNM system or the NR considers only one aspect of the LN status, which means the anatomic location or the relative number of metastatic LN. Few studies have investigated the combined effect of these two elements.

The Surveillance, Epidemiology and End Results (SEER) database is a national registry that collects cancer incidence and survival data and is representative of the US population (11). In this study, we used the SEER database to explore the prognostic value of the combined $\mathrm{pN}$ stage and NR (pN-NR) for NSCLC. We present the following article in accordance with the STROBE reporting checklist (available at http://dx.doi.org/10.21037/jtd-20-758).

\section{Methods}

\section{Data resource and study population}

Approval for this study and informed consent were waived from the ethics committee in the China-Japan Friendship Hospital due to the retrospective and public database nature of this study. Patients aged between 20 and 79 years old with $\mathrm{pN} 1$ or $\mathrm{pN} 2 \mathrm{NSCLC}$ who underwent radical resection (including lobectomy, bilobectomy, pneumonectomy and LN dissection) between 2004 and 2012 were retrieved from the SEER database using the SEER-Stat V8.3.5 in February 2019. The year 2004 was chosen because the 6th edition of the TNM classification was not uniformly available in the database until then, and by the time of our study the most recently released data were in 2012 . Patients who had small cell carcinoma, prior history of malignant tumor, pathological T0 or Tis disease, and stage IV disease were excluded from this study. Patients were also excluded whose number of examined $\mathrm{LN}$ was less than 6, who died within 30 days after surgery or without necessary clinicopathological information.

Patient records including age, gender, race, laterality, type of surgery, histological type, pathological stage, LN status, and follow-up information were obtained. The SEER registry provides detailed information regarding the pathological stage of $\mathrm{LN}$ involvement $(\mathrm{pN})$, the number of positive $\mathrm{LN}$ and the number of $\mathrm{LN}$ examined during surgery. The NR was divided into low and high group, and all patients were further divided into 4 categories with different combinations of $\mathrm{pN}$ and NR.

The primary outcome of interest was overall survival (OS), which was calculated using the interval between surgery and death of all causes or the end of this study; secondary outcome was cause-specific survival (CSS), which was defined as time to death from lung cancer, with patients censored at the end of this study.

\section{Statistical analysis}

All categorical variables were presented as a percentage. Continuous variables were reported as either mean and standard deviation (SD) or median and interquartile range (IQR) depending on the distribution of data. The maximally selected log-rank statistics was used for the analysis of NR to find the optimal cutoff with best discriminative ability of survival. This analysis was performed with every 0.05 increment of NR. The $\mathrm{pN}-\mathrm{NR}$ was investigated as the predictor of OS and CSS using Cox proportional hazards regression models (univariable and multivariable). Covariates included the following characteristics: age, race, gender, laterality, type of surgery, histological subtype, pTNM stage, $\mathrm{pT}$ stage, $\mathrm{pN}$ stage, $\mathrm{NR}$ and $\mathrm{pN}$ NR. A multivariable model was developed using stepwise regression (forwards selection) by selecting significant variables upon univariable analysis. Enter limit and remove limit were $\mathrm{P}=0.10$ and $\mathrm{P}=0.15$, respectively.

Survival curves were plotted using the Kaplan-Meier method and the log-rank test was used to assess differences between pN-NR groups. Survival was also estimated after stratified by age, $\mathrm{pT}$ stage, type of surgery, and histological type to assess if prognostic differences across the $4 \mathrm{pN}$ NR groups remained significant after controlling for these confounders. The results of all survival models were presented as adjusted hazard ratios (HR) with $95 \%$ confidence interval (95\% CI). Significance was defined by two-tailed $\mathrm{P}$ value $<0.05$. The SPSS software (IBM SPSS Statistics for Windows, Version 22.0. Armonk, NY: IBM Corp.) was used for all analyses. This study is based on the 
Table 1 Clinical characteristics and surgical outcomes

\begin{tabular}{|c|c|c|}
\hline Variables & Number of patients & $\%$ \\
\hline \multicolumn{3}{|l|}{ Age, years } \\
\hline$\leq 65$ & 5,847 & 48.0 \\
\hline$>65$ & 6,323 & 52.0 \\
\hline \multicolumn{3}{|l|}{ Gender } \\
\hline Male & 6,454 & 53.0 \\
\hline Female & 5,716 & 47.0 \\
\hline \multicolumn{3}{|l|}{ Race } \\
\hline Whites & 10,197 & 83.8 \\
\hline Blacks & 1,084 & 8.9 \\
\hline Others & 889 & 7.3 \\
\hline \multicolumn{3}{|l|}{ Laterality } \\
\hline Left & 5,765 & 47.4 \\
\hline Right & 6,405 & 52.6 \\
\hline \multicolumn{3}{|l|}{ Histology } \\
\hline Adenocarcinoma & 7,181 & 59.0 \\
\hline Squamous cell carcinoma & 3,757 & 30.9 \\
\hline Others & 1,232 & 10.1 \\
\hline \multicolumn{3}{|l|}{ Pathological stage } \\
\hline$\| A$ & 1,822 & 15.0 \\
\hline IIB & 3,970 & 32.6 \\
\hline IIIA & 4,956 & 40.7 \\
\hline IIIB & 1,422 & 11.7 \\
\hline \multicolumn{3}{|l|}{ Pathological T stage } \\
\hline $\mathrm{T} 1$ & 3,164 & 26.0 \\
\hline $\mathrm{T} 2$ & 6,770 & 55.6 \\
\hline T3 & 814 & 6.7 \\
\hline $\mathrm{T} 4$ & 1,422 & 11.7 \\
\hline \multicolumn{3}{|l|}{ Pathological N stage } \\
\hline N1 & 7,045 & 57.9 \\
\hline N2 & 5,125 & 42.1 \\
\hline \multicolumn{3}{|l|}{ Type of surgery } \\
\hline Lobectomy or bilobectomy & 10,240 & 84.1 \\
\hline Pneumonectomy & 1,930 & 15.9 \\
\hline
\end{tabular}

Strengthening the Reporting of Observational Studies in Epidemiology (STROBE) guideline (12). The statistical analysis is based on the European Journal of CardioThoracic Surgery and the Interactive Cardio Vascular and Thoracic Surgery guideline (13).

\section{Results}

From 2004 to 2012, 69,727 patients undergoing surgical resection for lung cancer with positive $\mathrm{LN}$ were identified from the SEER database. Among them 12,170 met the study criteria. Patient records including demographic, surgical and pathological information was presented in Table 1. The mean age of diagnosis was $64.8 \pm 9.2$ years old. Histological analysis showed a predominance of adenocarcinoma (59.0\%). The median numbers of resected LN and metastatic LN were 12 (IQR: 9-18) and 2 (IQR: 1-4), respectively. N1 and N2 LN metastasis were identified in $57.9 \%$ and $42.1 \%$ cases respectively.

A total of 6,621 (54.4\%) deaths were recorded by the time of this study and 5,039 (41.4\%) deaths were caused by lung cancer. The 5 -year OS and CSS were $41.7 \%$ and $50.7 \%$. As for the relationship between NR and OS or CSS, the maximal $\chi^{2}$ value was reached when the NR setting was 0.3. So the NR was divided into low and high groups with this cut point, and all patients were further divided into 4 groups: $\mathrm{pN} 1-\mathrm{NR}<0.3, \mathrm{pN} 1-\mathrm{NR} \geq 0.3, \mathrm{pN} 2-\mathrm{NR}<0.3$ and $\mathrm{pN} 2-\mathrm{NR} \geq 0.3$.

In univariable analysis, age, gender, race, surgery type, histological type, pTNM stage, $\mathrm{pT}$ stage, $\mathrm{pN}$ stage, NR and $\mathrm{pN}-\mathrm{NR}$ were predictive factors for both OS and CSS. In multivariable analysis, age, gender, race, surgery type, histological type, $\mathrm{pT}$ stage and $\mathrm{pN}-\mathrm{NR}$ were independent prognostic factors for OS, and age, gender, surgery type, histological type, $\mathrm{pT}$ stage and $\mathrm{pN}-\mathrm{NR}$ were independent prognostic factors for CSS (Table 2). Compared with those for the patients in the $\mathrm{pN} 1-\mathrm{NR}<0.3$ group, the hazard ratio of OS was 1.405 (95\% CI: $1.295-1.524), 1.183$ (95\% CI: $1.113-1.257)$ and 1.717 (95\% CI: $1.607-1.835)$ times higher for patients in the $\mathrm{pN} 1-\mathrm{NR} \geq 0.3$, $\mathrm{pN} 2-\mathrm{NR}<0.3$ and pN2-NR $\geq 0.3$ groups, respectively, and 1.518 (95\% CI: 1.385-1.665), 1.221 (95\% CI: 1.138-1.310), 1.849 (95\% CI: 1.715-1.993) times higher for CSS (Table 2).

The survival curves showed that $\mathrm{NR}<0.3$ was associated 
Table 2 Multivariable analysis for OS and CSS

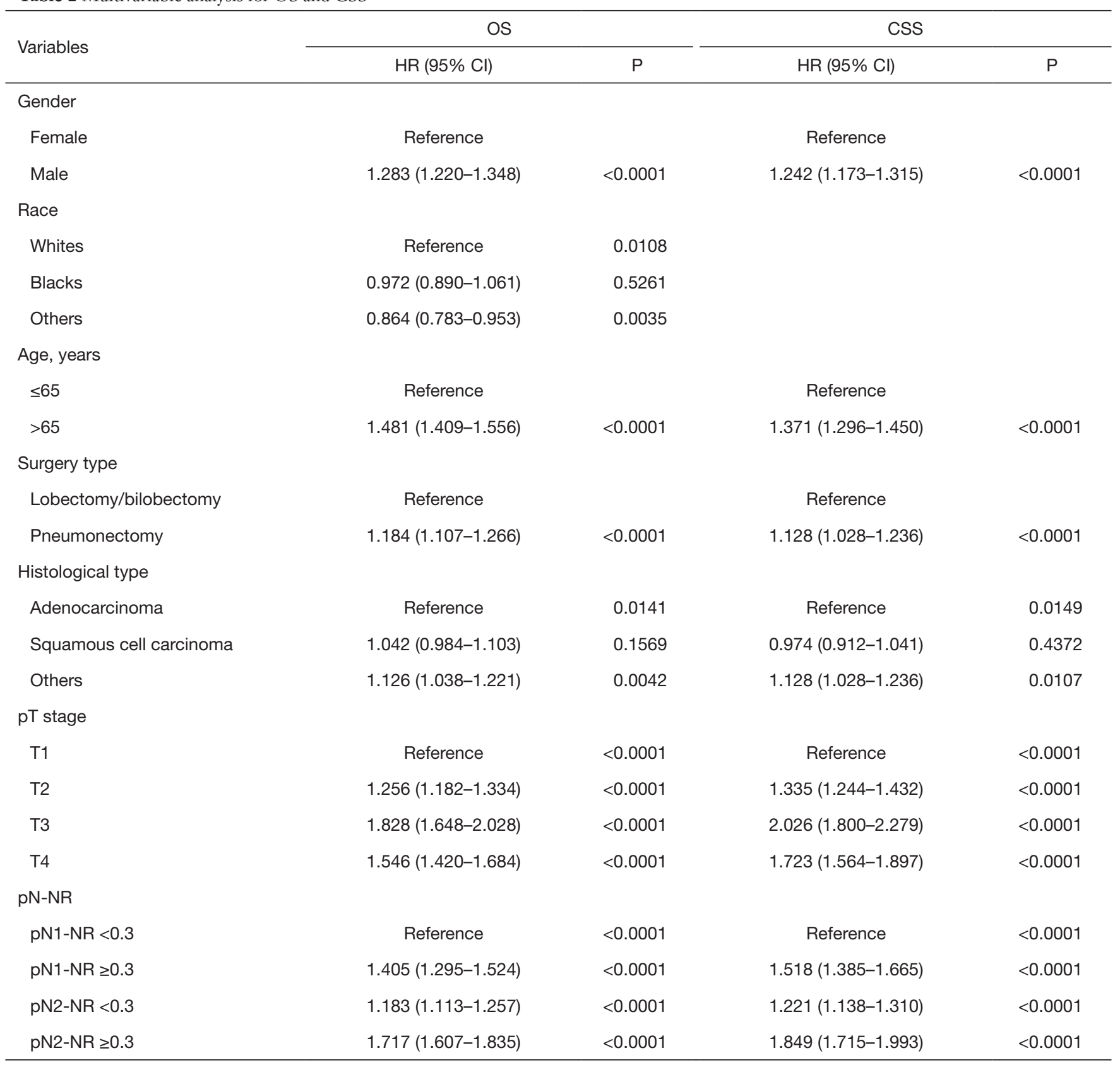

OS, overall survival; CSS, cause-specific survival; HR, hazard ratio; $\mathrm{Cl}$, confidence interval; NR, node ratio.

with better survival in the entire population, with 5 -year OS $45.6 \%$ vs. $31.0 \%(\mathrm{P}<0.0001)$ and 5 -year CSS $55.1 \%$ vs. $38.1 \%(\mathrm{P}<0.0001)$ (Figure 1). When stratified by the pN-NR, the survival curves separated well between the 4 groups, with 5 -year OS $47.1 \%$ for $\mathrm{pN} 1-\mathrm{NR}<0.3,43.0 \%$ for $\mathrm{pN} 2-\mathrm{NR}<0.3,35.0 \%$ for $\mathrm{pN} 1-\mathrm{NR} \geq 0.3$ and $28.5 \%$ for $\mathrm{pN} 2-\mathrm{NR} \geq 0.3$. The differences in survival between neighboring pN-NR groups were statistically significant. The same trend went for CSS, with 5 -year survival rate $56.8 \%$ for $\mathrm{pN} 1-\mathrm{NR}<0.3,51.7 \%$ for $\mathrm{pN} 2-\mathrm{NR}<0.3,43.3 \%$ for $\mathrm{pN} 1-\mathrm{NR} \geq 0.3$ and $35.6 \%$ for $\mathrm{pN} 2-\mathrm{NR} \geq 0.3$ (Figure 2).

The prognostic role of $\mathrm{pN}-\mathrm{NR}$ was further analyzed within subgroups. For patients with T1/T2 disease, adenocarcinoma, age $<65$ or $\geq 65$, and lobectomy/ 
Table 3 Five-year OS and CSS for patients in different subgroups

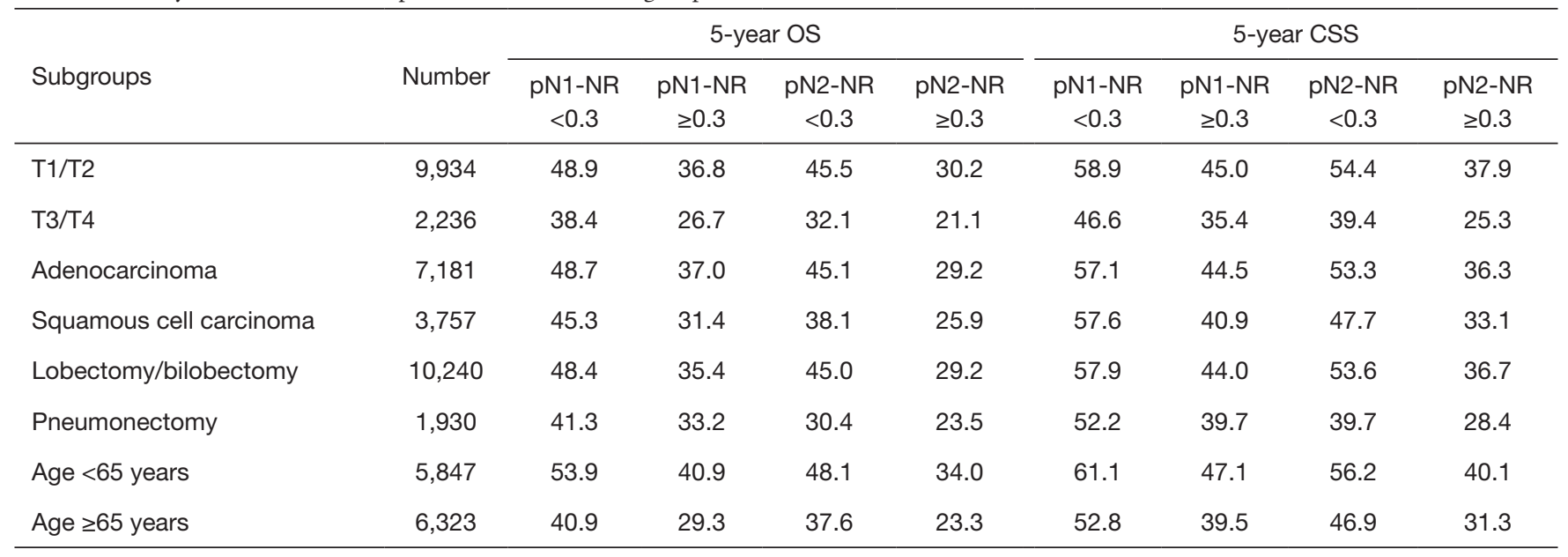

OS, overall survival, CSS, cause-specific survival; NR, node ratio.
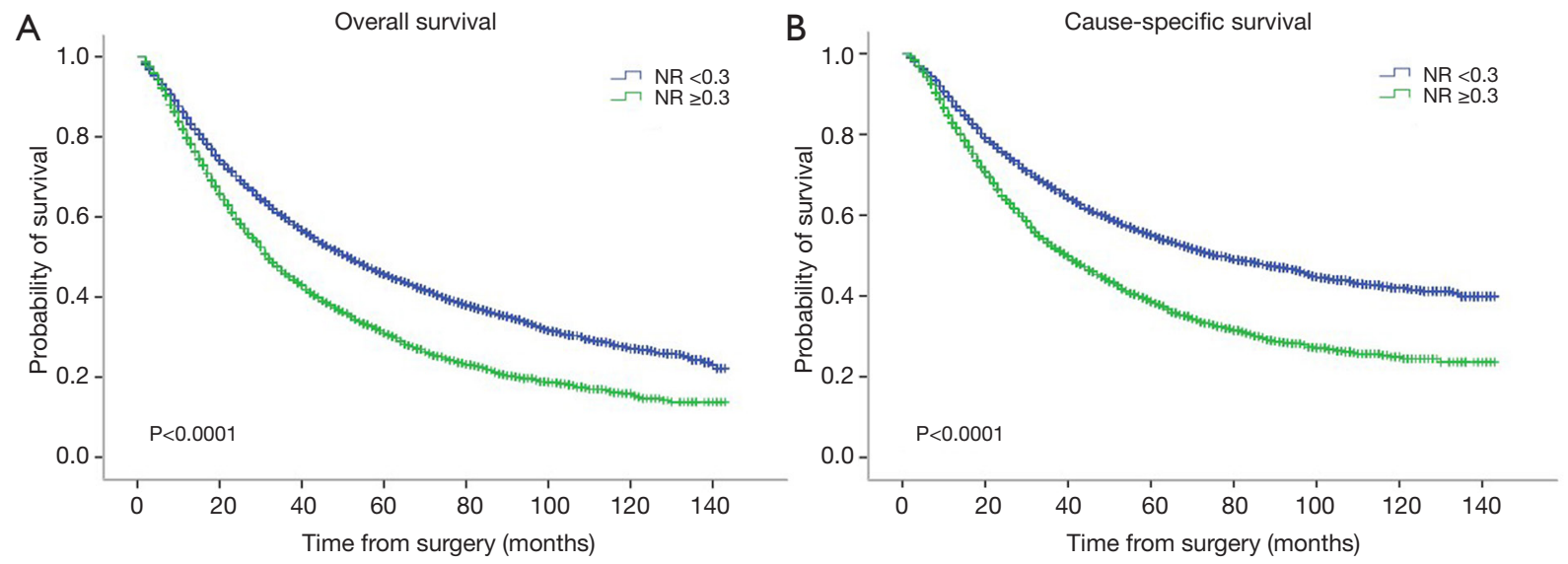

Figure 1 OS (A) and CSS (B) according to NR in the entire population. OS, overall survival, CSS, cause-specific survival; NR, node ratio.
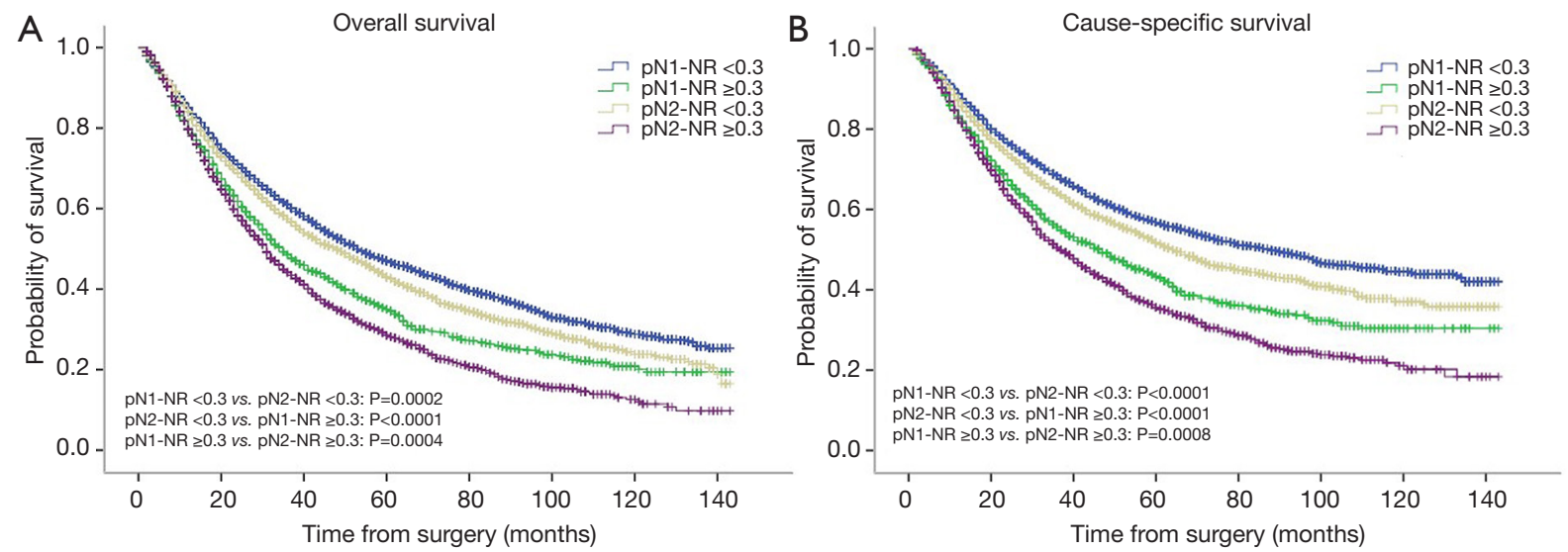

Figure 2 OS (A) and CSS (B) according to pN-NR in the entire population. OS, overall survival, CSS, cause-specific survival; NR, node ratio. 

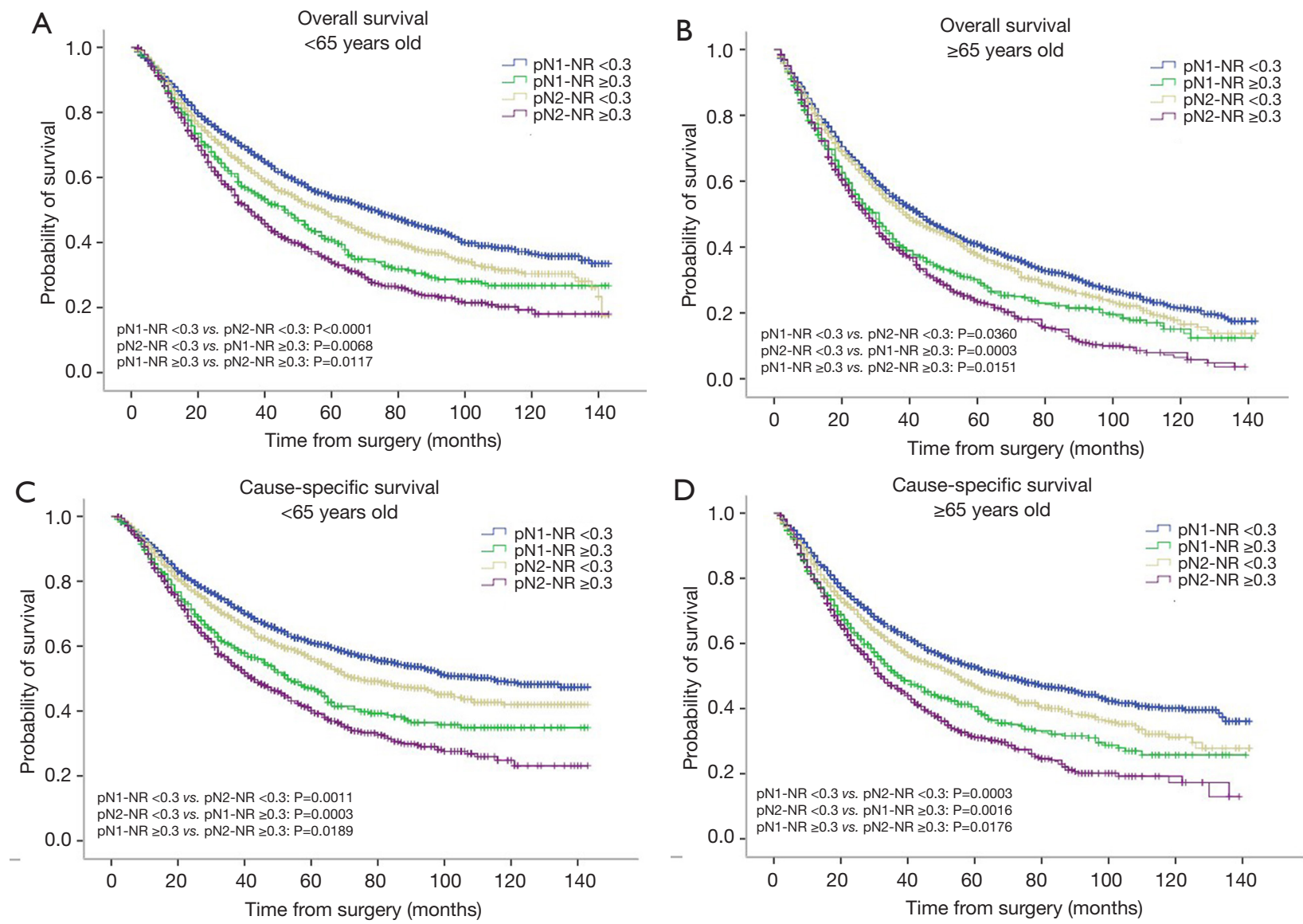

Figure 3 OS (A,B) and CSS (C,D) according to pN-NR in different age groups. OS, overall survival, CSS, cause-specific survival; NR, node ratio.

bilobectomy, the best OS and CSS were reached in the pN1-NR $<0.3$ group, followed by the $\mathrm{pN} 2-\mathrm{NR}<0.3$ group, the $\mathrm{pN} 1-\mathrm{NR} \geq 0.3$ group, and the $\mathrm{pN} 2-\mathrm{NR} \geq 0.3$ group in sequence, and the differences between neighboring $\mathrm{pN}-\mathrm{NR}$ groups were statistically significant. For patients with T3/ $\mathrm{T} 4$ disease, squamous cell carcinoma and pneumonectomy, the survival of the $\mathrm{pN} 2-\mathrm{NR}<0.3$ group and the $\mathrm{pN} 1-\mathrm{NR}$ $\geq 0.3$ group wasn't statistically significant, though the pN2NR $<0.3$ group was better except for pneumonectomy (Figures 3,4,5,6, Table 3).

\section{Discussion}

The pathological status of $\mathrm{LN}$ is one of the most important prognostic factors for NSCLC after surgery. In the current TNM system, the assessment of nodal status has always only focused on the anatomic location of metastatic LN (3). However, some studies have shown that this nodal staging method is unsatisfactory in distinguishing the heterogeneous $\mathrm{pN} 1$ and $\mathrm{pN} 2 \mathrm{NSCLC}(2,14)$. As a result, some authors suggested that NR, which takes into account the number of resected $\mathrm{LN}$ and positive $\mathrm{LN}$, could be a useful predictive factor for survival $(7,15,16)$. In this study we used the population-based SEER database to assess the combined efficiency of node location and node ratio $(\mathrm{pN}$ NR) for the prognosis of NSCLC after surgery.

In this study, patients were excluded whose number of resected LN was less than 6 to ensure relatively standard lymphadenectomy. This limit is recommended by the European Society of Thoracic Surgeons (17), and some other studies also used this limit $(9,10)$. The value of NR could be significantly affected by extremums if only few or no LN was harvested. Another consideration is that there is evidence showing that inadequate $\mathrm{LN}$ resection is an independent factor for poorer survival, and we tried to eliminate this effect $(6,18)$. Some studies also suggested 10 $\mathrm{LN}$, even $16 \mathrm{LN}$ for accurate assessment of nodal status $(6,14)$. To keep a balance between the quality of surgery and 

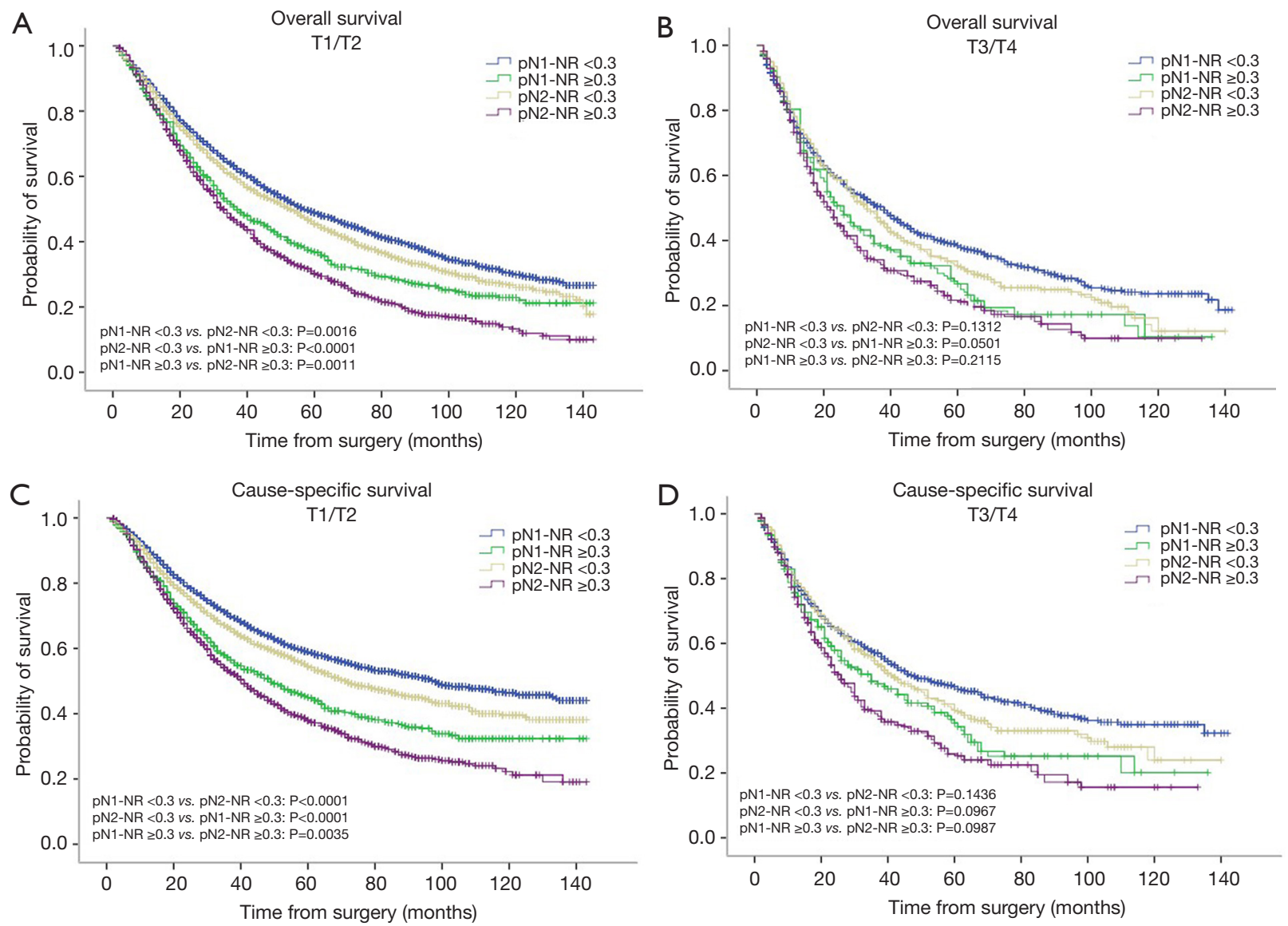

Figure 4 OS (A,B) and CSS (C,D) according to pN-NR in different pT stages. OS, overall survival, CSS, cause-specific survival; NR, node ratio.

study sample size, we set resection of $6 \mathrm{LN}$ as the threshold for inclusion. The median number of resected $\mathrm{LN}$ in our study was 12 . Actually, 45,047 cases were excluded for this single criterion in our study. So, we believe this criterion is crucial to ensure the quality of this study. Patients with pN0 were also excluded because it's impossible to calculate the NR in these patients, and it is widely accepted that these patients have better survival than node positive patients (3). In this study we are unable to distinguish systemic LN dissection and LN sampling. However, since all the patients in this study were node positive and had at least $6 \mathrm{LN}$ harvested, we believe that they received relatively sufficient $\mathrm{LN}$ dissection.

Some authors have investigated the effect of NR on survival for lung cancer, and the cut point of NR they determined ranged between $15-50 \%(15,19-21)$. There are two studies based on the SEER database and both have drawn the conclusion that a lower NR is associated with better survival $(20,22)$. But these two studies didn't restrict the minimum number of $\mathrm{LN}$ resected, and both collected data from a long-time span (1988 to 2007), which might result in significant patient selection bias. The management of lung cancer, including the preoperative staging method, neoadjuvant therapy and the extent of mediastinal lymphadenectomy could be different in the 1980s and 1990s. So, their study population was highly heterogeneous, and included a number of cases without adequate LN assessment. What's more, the cut point of NR they chose was simply determined by the mathematical distribution of $\mathrm{NR}$, and thus could result in significant bias. In our study, the cases selected were between 2004 to 2012 and with at least $6 \mathrm{LN}$ resected. What's more, we used the maximally selected log-rank statistics to find out the optimal cut point of NR, and thus we believe our result is more convincing.

The $\mathrm{pN}-\mathrm{NR}$ evaluated in this study takes into consideration two important aspects of the status of metastasis LN, which are the anatomic location and the positive $\mathrm{LN}$ ratio. We found out that the survival curves 

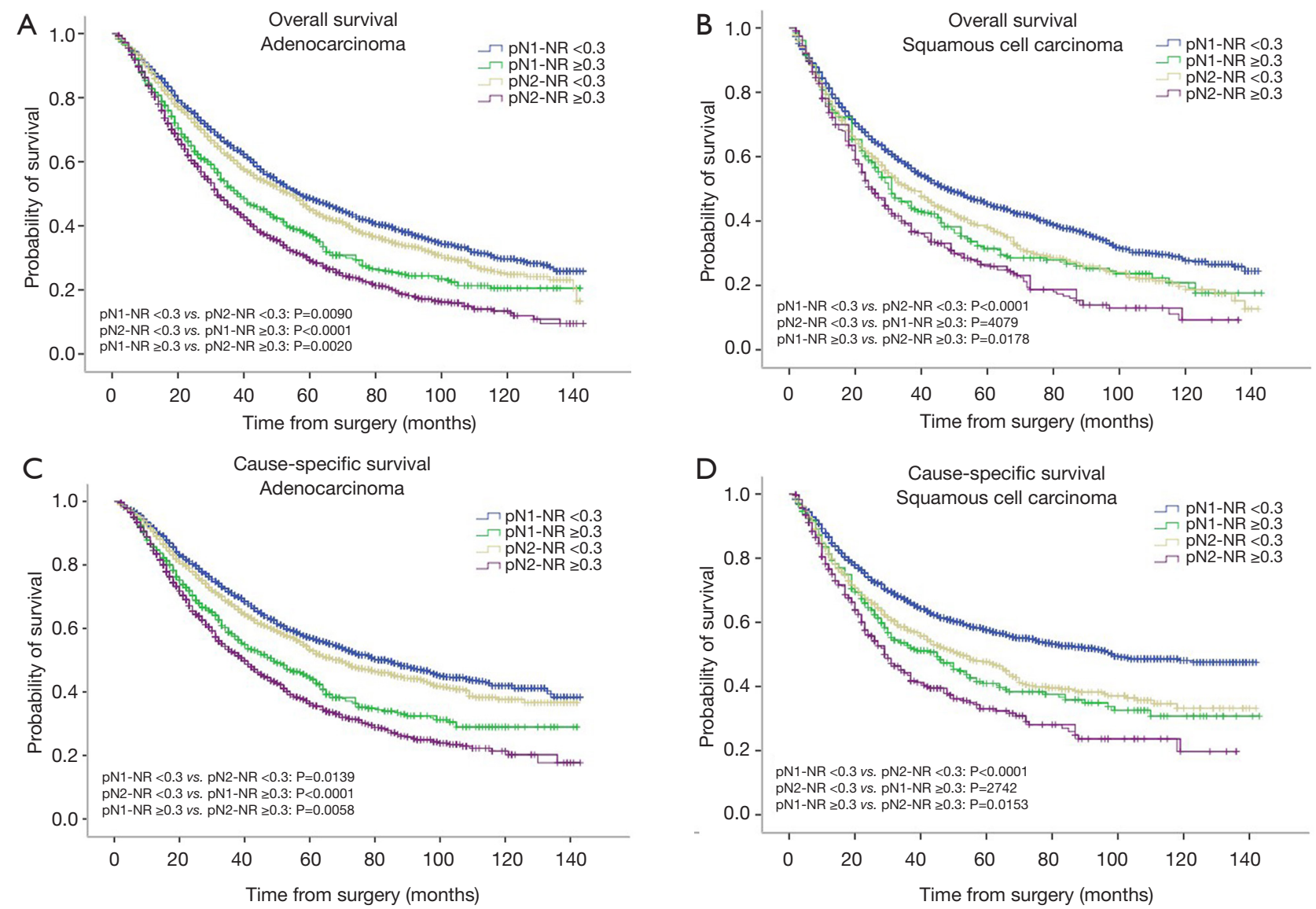

Figure 5 OS (A,B) and CSS (C,D) according to pN-NR in different histological types. OS, overall survival, CSS, cause-specific survival; NR, node ratio.

of the different $\mathrm{pN}-\mathrm{NR}$ groups separated well with each other for both OS and CSS, and this result was consistent in most subgroups. This means that the $\mathrm{pN}-\mathrm{NR}$ has a powerful discriminative ability concerning the prognosis of NSCLC. So, we believe it could be an eligible index for the description of nodal status for NSCLC after surgery. What's more, the pN-NR is easy to calculate and obtain.

The survival curves of the different $\mathrm{pN}-\mathrm{NR}$ groups were not statistically significant in the T3/T4 subgroup, though a tendency towards the deterioration of OS and CSS was similar to that of the entire population. The reason may be that the prognosis of the higher $\mathrm{pT}$ category is already poor, regardless of the status of metastatic LNs. What's more, the pT staging of the 6th edition used in this study is quite different from that of the current $8^{\text {th }}$ edition, so the effectiveness of $\mathrm{pN}-\mathrm{NR}$ for higher $\mathrm{pT}$ stage should be explored with the latest system (23). In patients undergoing pneumonectomy, the survival curves overlapped. This may be related to the surgery itself. Pneumonectomy is a major surgery, and the survival benefit for tumor resection could be significantly affected by the comorbidity of patients, while the node status is less important (24). But we are unable to obtain this information from the SEER database.

In this study, the patients in the $\mathrm{pN} 2-\mathrm{NR}<0.3$ group had better OS and CSS than the patients in the pN1-NR $\geq 0.3$ group, and subgroup analysis supported this finding in most occasions though in some cases the difference wasn't statistically significant. It's widely accepted that the pN2 patient group has worse prognosis than the $\mathrm{pN} 1$ patient group when other conditions are comparable. A Japanese investigation showed that the 5 -year OS for $\mathrm{pN} 1$ and $\mathrm{pN} 2$ were $65.9 \%$ and $35.4 \%$, respectively; the 5 -year DFS for $\mathrm{pN} 1$ and $\mathrm{pN} 2$ were $75.3 \%$ and $31.1 \%$, respectively (14). But our result indicated that the prognosis of $\mathrm{pN} 1$ and $\mathrm{pN} 2$ could be reversed when stratified by the pN-NR. This reflects a possible limitation of the present $\mathrm{pN}$ classification system for nodal status. There are several possible underlying reasons for this phenomenon. First, the NR 

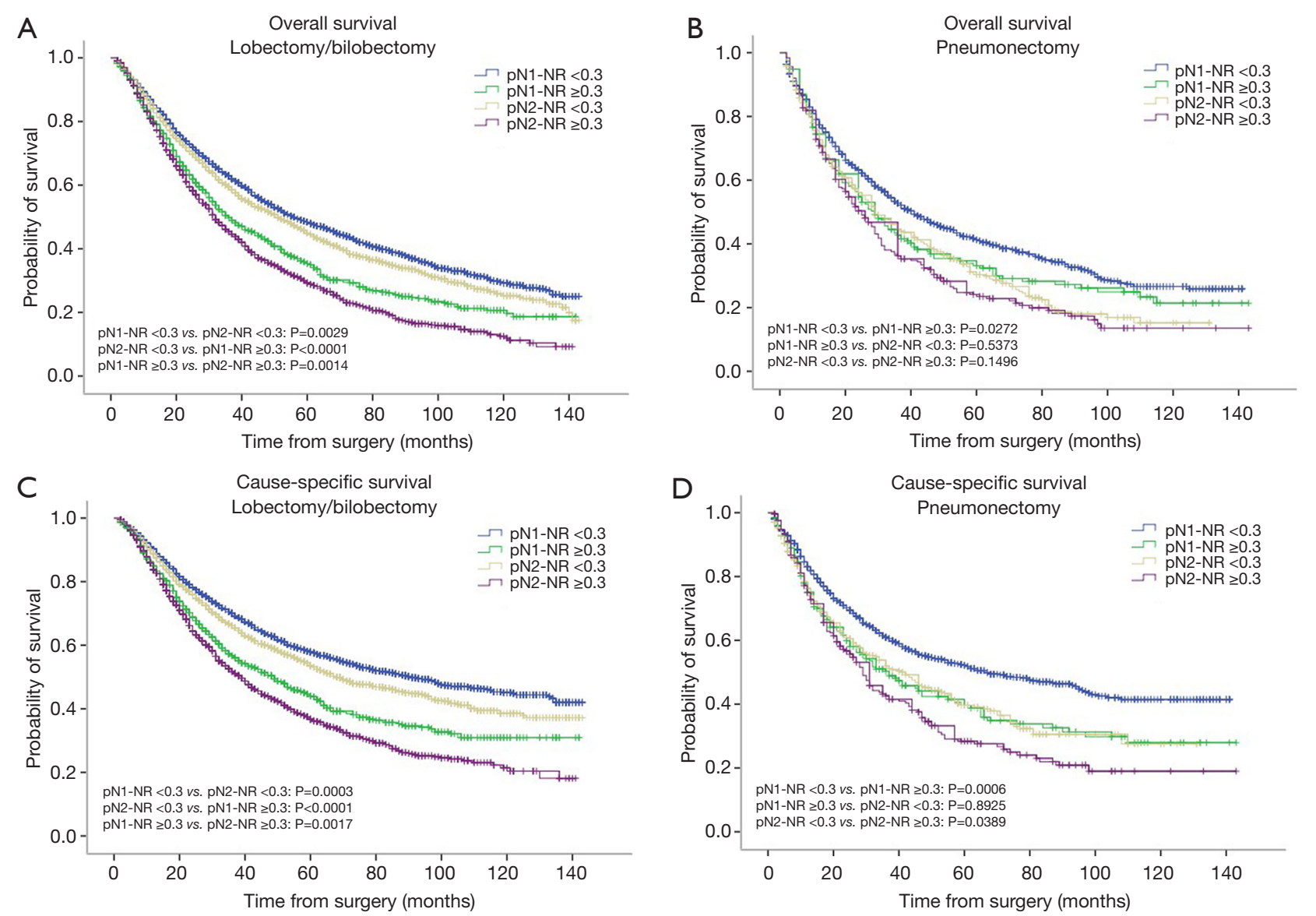

Figure 6 OS (A,B) and CSS (C,D) according to pN-NR in different surgery types. OS, overall survival, CSS, cause-specific survival; NR, node ratio.

seems to be a more powerful factor for survival. A higher NR could indicate a more aggressive malignant behavior, or higher LN tumor burden, which can exceed the effect of metastatic LN station. Several studies have a similar finding. In the study by Ding et al. involving 700 node positive NSCLC patients, the NR was a superior prognostic factor (8). A Japanese research involving $437 \mathrm{pN} 0,113 \mathrm{pN} 1$ and $101 \mathrm{pN} 2$ patients reported that LNR followed by $\mathrm{nN}$ may be a more effective prognostic indicator than $\mathrm{pN}(25)$. Second, the patients in the $\mathrm{pN} 1-\mathrm{NR} \geq 0.3$ group may be understaged to $\mathrm{pN} 1$ due to insufficient lymphadenectomy. The correlation between the number of examined LN and stage migration has been reported (6). Since all patients in this study were node positive, it's possible that a relatively high NR in the $\mathrm{pN} 1-\mathrm{NR} \geq 0.3$ group could result from a relatively small number of resected $\mathrm{LN}$, thus potential positive N2 could be missed out. The undiscovered N2 disease may discourage patients from receiving proper adjuvant therapy and active follow-up, and thus result in compromised OS and CSS. Another study also proved that a small number of resected LN is directly associated with a worse survival outcome in NSCLC patients (26). Finally, the patients in the $\mathrm{pN} 2-\mathrm{NR}<0.3$ group are more likely to have lobe-specific metastatic or skip-N2 disease, or singlestation N2 disease, which was reported to have better survival than pN2 disease in general (27-29). This also reflects that N2 is a highly heterogeneous disease group which needs to be further stratified under the current TNM system, and the $\mathrm{pN}-\mathrm{NR}$ could be a promising index.

The NR has been used to stratify patients with different recurrent risks and guide treatment (30). We believe that the pN-NR could also be used to guide treatment. A study in 2016 using the National Cancer Database found out that only $53 \%$ of the $\mathrm{pN} 1$ patients received peri-operative chemotherapy though it is recommended by established guidelines (31). This may be due to the conception that $\mathrm{pN} 1$ patients have better survival than $\mathrm{pN} 2$ patients, and the concerns about the severe adverse effects of chemotherapy. 
But according to our result, for the $\mathrm{pN} 1$ patients with NR $\geq 0.3$, the OS and CSS were worse than the $\mathrm{pN} 2$ patients with $\mathrm{NR}<0.3$. According to this result, maybe aggressive adjuvant therapy should be considered for $\mathrm{pN} 1-\mathrm{NR} \geq 0.3$ patients, and this needs to be further explored.

The limitations of this study are attributable to the retrospective nature and the inherent limitations from using the SEER database. The SEER database lacks granular details such as smoking history, comorbidity, clinical staging, peri-operative therapy, LN station dissected and recurrence patterns (32). So, the results of this study should not be overinterpreted given the inherent weaknesses in SEER analyses in general. Neoadjuvant chemotherapy could lead to LN downstage. However, according to the EMERGING-CTONG 1103 trial, the LN down-stage occurred in only $2.9 \%$ patients in the neoadjuvant chemotherapy group (33). So, we believe that the pre-operative chemotherapy could have very limited effect on our result. Another relevant point is that we collected cases over a long time period, and the surgical techniques regarding $\mathrm{LN}$ dissection could be different in recent years compared with those of earlier times. In addition, two possible biases could lead to a miscount of the $\mathrm{LN}$ number: underestimation as a result of the difficulty in separating each $\mathrm{LN}$ in the dissected tissues and overestimation because of fragmentation of nodal tissues during the removal of LNs. All these factors could complicate the interpretation of results. What's more, right now it is impossible to accurately estimate the number of metastatic LN for both pre-operative and inoperable NSCLC through any diagnostic methods, so the pN-NR could only be applied for the p stage.

\section{Conclusions}

This study reveals that the pN-NR could be a good predictor for the prognosis of NSCLC after curative resection and 0.3 is the optimal cut point of NR. The finding has the potential to act as a useful supplement to the current TNM staging system and guide adjuvant therapy. Prospective studies are needed to validate the effectiveness of $\mathrm{pN}-\mathrm{NR}$ classification for NSCLC in the future.

\section{Acknowledgments}

Funding: None.

\section{Footnote}

Reporting Checklist: The authors have completed the
STROBE reporting checklist. Available at http://dx.doi. org/10.21037/jtd-20-758

Peer Review File: Available at http://dx.doi.org/10.21037/jtd20-758

Conflicts of Interest: All authors have completed the ICMJE uniform disclosure form (available at http://dx.doi. org/10.21037/jtd-20-758). The authors have no conflicts of interest to declare.

Ethical Statement: The authors are accountable for all aspects of the work in ensuring that questions related to the accuracy or integrity of any part of the work are appropriately investigated and resolved. The study was conducted in accordance with the Declaration of Helsinki (as revised in 2013). Approval for this study and informed consent were waived from the ethics committee in the China-Japan Friendship Hospital due to the retrospective and public database nature of this study.

Open Access Statement: This is an Open Access article distributed in accordance with the Creative Commons Attribution-NonCommercial-NoDerivs 4.0 International License (CC BY-NC-ND 4.0), which permits the noncommercial replication and distribution of the article with the strict proviso that no changes or edits are made and the original work is properly cited (including links to both the formal publication through the relevant DOI and the license). See: https://creativecommons.org/licenses/by-nc-nd/4.0/.

\section{References}

1. Bray F, Ferlay J, Soerjomataram I, et al. Global cancer statistics 2018: GLOBOCAN estimates of incidence and mortality worldwide for 36 cancers in 185 countries. CA Cancer J Clin 2018;68:394-424.

2. Wei S, Asamura H, Kawachi R, et al. Which is the better prognostic factor for resected non-small cell lung cancer: the number of metastatic lymph nodes or the currently used nodal stage classification? J Thorac Oncol 2011;6:310-8.

3. Asamura H, Chansky K, Crowley J, et al. The International Association for the Study of Lung Cancer Lung Cancer Staging Project: Proposals for the Revision of the N Descriptors in the Forthcoming 8th Edition of the TNM Classification for Lung Cancer. J Thorac Oncol 2015;10:1675-84. 
4. Donohoe CL, Phillips AW. Cancer of the esophagus and esophagogastric junction: an 8(th) edition staging primer. J Thorac Dis 2017;9:E282-4.

5. Giuliano AE, Connolly JL, Edge SB, et al. Breast CancerMajor changes in the American Joint Committee on Cancer eighth edition cancer staging manual. CA Cancer J Clin 2017;67:290-303.

6. Liang W, He J, Shen Y, et al. Impact of Examined Lymph Node Count on Precise Staging and Long-Term Survival of Resected Non-Small-Cell Lung Cancer: A Population Study of the US SEER Database and a Chinese MultiInstitutional Registry. J Clin Oncol 2017;35:1162-70.

7. Taylor MD, LaPar DJ, Thomas CJ, et al. Lymph node ratio predicts recurrence and survival after R0 resection for non-small cell lung cancer. Ann Thorac Surg 2013;96:1163-70.

8. Ding X, Hui Z, Dai H, et al. A Proposal for Combination of Lymph Node Ratio and Anatomic Location of Involved Lymph Nodes for Nodal Classification in Non-Small Cell Lung Cancer. J Thorac Oncol 2016;11:1565-73.

9. Pawełczyk K, Marciniak M, Blasiak P. Evaluation of new classifications of $\mathrm{N}$ descriptor in non-small cell lung cancer (NSCLC) based on the number and the ratio of metastatic lymph nodes. J Cardiothorac Surg 2016;11:68.

10. Chiappetta M, Leuzzi G, Sperduti I, et al. Lymph-node ratio predicts survival among the different stages of nonsmall-cell lung cancer: a multicentre analysis. Eur J Cardiothorac Surg 2019;55:405-12.

11. Cronin KA, Ries LA, Edwards BK. The Surveillance, Epidemiology, and End Results (SEER) Program of the National Cancer Institute. Cancer 2014;120 Suppl 23:3755-7.

12. von Elm E, Altman DG, Egger M, et al. The Strengthening the Reporting of Observational Studies in Epidemiology (STROBE) Statement: guidelines for reporting observational studies. Int J Surg 2014;12:1495-9.

13. Hickey GL, Dunning J, Seifert B, et al. Statistical and data reporting guidelines for the European Journal of Cardio-Thoracic Surgery and the Interactive CardioVascular and Thoracic Surgery. Eur J Cardiothorac Surg 2015;48:180-93.

14. Saji H, Tsuboi M, Shimada Y, et al. A proposal for combination of total number and anatomical location of involved lymph nodes for nodal classification in non-small cell lung cancer. Chest 2013;143:1618-25.

15. Qiu C, Dong W, Su B, et al. The prognostic value of ratiobased lymph node staging in resected non-small-cell lung cancer. J Thorac Oncol 2013;8:429-35.
16. Kang CH, Ra YJ, Kim YT, et al. The impact of multiple metastatic nodal stations on survival in patients with resectable N1 and N2 nonsmall-cell lung cancer. Ann Thorac Surg 2008;86:1092-7.

17. De Leyn P, Lardinois D, Van Schil P, et al. European trends in preoperative and intraoperative nodal staging: ESTS guidelines. J Thorac Oncol 2007;2:357-61.

18. Fukui T, Mori S, Yokoi K, et al. Significance of the number of positive lymph nodes in resected non-small cell lung cancer. J Thorac Oncol 2006;1:120-5.

19. Wang CL, Li Y, Yue DS, et al. Value of the metastatic lymph node ratio for predicting the prognosis of non-small-cell lung cancer patients. World J Surg 2012;36:455-62.

20. Nwogu CE, Groman A, Fahey D, et al. Number of lymph nodes and metastatic lymph node ratio are associated with survival in lung cancer. Ann Thorac Surg 2012;93:1614-9; discussion 1619-20.

21. Wisnivesky JP, Arciniega J, Mhango G, et al. Lymph node ratio as a prognostic factor in elderly patients with pathological N1 non-small cell lung cancer. Thorax 2011;66:287-93.

22. Jonnalagadda S, Arcinega J, Smith C, et al. Validation of the lymph node ratio as a prognostic factor in patients with N1 nonsmall cell lung cancer. Cancer 2011;117:4724-31.

23. Goldstraw P, Chansky K, Crowley J, et al. The IASLC Lung Cancer Staging Project: Proposals for Revision of the TNM Stage Groupings in the Forthcoming (Eighth) Edition of the TNM Classification for Lung Cancer. J Thorac Oncol 2016;11:39-51.

24. Doddoli C, Barlesi F, Trousse D, et al. One hundred consecutive pneumonectomies after induction therapy for non-small cell lung cancer: an uncertain balance between risks and benefits. J Thorac Cardiovasc Surg 2005;130:416-25.

25. Matsuguma H, Oki I, Nakahara R, et al. Proposal of new nodal classifications for non-small-cell lung cancer based on the number and ratio of metastatic lymph nodes. Eur J Cardiothorac Surg 2012;41:19-24.

26. Wen YS, Xi KX, Xi KX, et al. The number of resected lymph nodes is associated with the long-term survival outcome in patients with T2 N0 non-small cell lung cancer. Cancer Manag Res 2018;10:6869-77.

27. Li H, Hu H, Wang R, et al. Lung adenocarcinoma: Are skip N2 metastases different from non-skip? J Thorac Cardiovasc Surg 2015;150:790-5.

28. Sun Y, Gao W, Zheng H, et al. Mediastinal lymph-nodes metastasis beyond the lobe-specific: an independent risk 
factor toward worse prognoses. Ann Thorac Cardiovasc Surg 2014;20:284-91.

29. Wang L, Zhan C, Gu J, et al. Role of Skip Mediastinal Lymph Node Metastasis for Patients With Resectable Non-small-cell Lung Cancer: A Propensity Score Matching Analysis. Clin Lung Cancer 2019;20:e346-55.

30. Shang X, Li Z, Lin J, et al. PLNR $\leq 20 \%$ may be a benefit from PORT for patients with IIIA-N2 NSCLC: a large population-based study. Cancer Manag Res 2018;10:3561-7.

31. Bott MJ, Patel AP, Verma V, et al. Patterns of care in

Cite this article as: Xing $\mathrm{H}, \mathrm{Hu} \mathrm{M}$, Chen J, Guo Y, Liu D, Liang C. Combining node location and node ratio as a prognostic factor for surgical resected non-small cell lung cancer: a population-based study. J Thorac Dis 2020;12(7):35493560. doi: $10.21037 /$ jtd-20-758 hilar node-positive (N1) non-small cell lung cancer: A missed treatment opportunity? J Thorac Cardiovasc Surg 2016;151:1549-58.e2.

32. Varlotto JM, Recht A, Nikolov M, et al. Extent of lymphadenectomy and outcome for patients with stage I nonsmall cell lung cancer. Cancer 2009;115:851-8.

33. Zhong WZ, Chen KN, Chen C, et al. Erlotinib Versus Gemcitabine Plus Cisplatin as Neoadjuvant Treatment of Stage IIIA-N2 EGFR-Mutant Non-Small-Cell Lung Cancer (EMERGING-CTONG 1103): A Randomized Phase II Study. J Clin Oncol 2019;37:2235-45. 